\title{
Persuasives Handeln im wissenschaftlichen Diskurs und seine lexikografische Darstellung: das Beispiel der Kollokation Bild zeichnen
}

\section{Einleitung}

Persuasives Handeln als ein auf die Überzeugung eines Adressaten gerichtetes Sprachhandeln gestaltet sich je nach Sprachverwendungsbereich unterschiedlich. Während in Bereichen wie Politik und Wirtschaft augenscheinlich persuasive Handlungszusammenhänge vorliegen, erschließt sich der persuasive Charakter von Wissenschaftskommunikation nicht unmittelbar. Dennoch ist sprachliches Handeln in der Wissenschaft deutlich geprägt durch die Notwendigkeit, die Rezipientengemeinschaft vom Wert der eigenen wissenschaftlichen Leistung $\mathrm{zu}$ überzeugen. Dieser Aspekt ist vor allem im Hinblick auf die Aneignung wissenschaftssprachlicher Kompetenzen relevant. Eine adäquate wissenschaftssprachliche Handlungsfähigkeit setzt die Kenntnis der sprachlichen Mittel und ihrer Gebrauchskonventionen voraus, die einem Text einen persuasiven Charakter verleihen. Um die Aneignung wissenschaftssprachlicher Kompetenz zu unterstützen, ist daher eine lexikografische Darstellung entsprechender Ausdrucksmittel erforderlich, die den grundlegenden persuasiven Charakter von Wissenschaftskommunikation berücksichtigt. Doch wie lässt sich die sprachliche Umsetzung des auf die Überzeugung gerichteten Handelns in der Wissenschaftskommunikation lexikografisch darstellen?

Der vorliegende Beitrag geht dieser Frage für das Deutsche exemplarisch anhand der durch die Kollokation Bild zeichnen realisierten Handlungen nach. Die hierzu vorgestellte Untersuchung erfolgte im Rahmen eines lexikografischen Projekts zur Erschließung der allgemeinen Wissenschaftssprache der Geisteswissenschaften. 
Der Beitrag gliedert sich wie folgt: Zunächst wird Wissenschaftskommunikation als persuasiv geprägtes und kontroversenorientiertes Handlungsfeld umrissen. Da das auf Überzeugung gerichtete Sprachhandeln in der Wissenschaft nicht auf Einzelwortebene erfassbar ist, sondern sich erst in komplexeren Strukturen manifestiert, sind im Hinblick auf die lexikografische Beschreibung Wortverbindungskonzepte wie das der Kollokationen heranzuziehen. Daher wird im zweiten Teil das Konzept der Kollokationen als mögliche Repräsentationsform allgemeinwissenschaftssprachlicher Handlungen näher betrachtet. Im dritten Teil wird eine Korpusstudie vorgestellt, welche die Verwendung der Kollokation Bild zeichnen im wissenschaftlichen Diskurs nachzeichnet. Dabei wird zunächst das Spektrum an sprachlichen Handlungen ermittelt, die mit Hilfe der Kollokation realisiert werden. Im Anschluss werden qualifizierende Verwendungen der Kollokation fokussiert, anhand derer die sprachliche Umsetzung des auf Überzeugung gerichteten Handelns in der Wissenschaftskommunikation exemplifiziert werden kann. Auf der Basis der Ergebnisse wird abschließend anhand des Beispiels Bild zeichnen skizziert, wie sich die sprachliche Umsetzung des persuasiven Charakters von Wissenschaftskommunikation in eine lexikografische Aufbereitung einbinden ließe.

\section{Wissenschaftskommunikation als persuasiv geprägtes und kontroversenorientiertes Handlungsfeld}

Die Wissenschaftssprachforschung untersucht sprachliche Mittel im Hinblick auf ihre Funktionalität zur Realisierung sprachlicher Handlungen im Handlungsfeld der Wissenschaft. Sie betrachtet damit die Sprachverwendung, durch die sich die wissenschaftliche Kommunikationsgemeinschaft als gesellschaftliche Institution maßgeblich konstituiert (vgl. Kretzenbacher 1998:134). Grundlegend für dieses Handlungsfeld ist dabei, dass wissenschaftliche Erkenntnisse erst durch die sprachliche Mitteilung in Form der Veröffentlichung für die Wissenschaftsgemeinschaft Relevanz gewinnen. Daraus ergibt sich für wissenschaftliche Veröffentlichungen (seien es Vorträge, Poster, Zeitschriftenbeiträge oder Monografien) die Notwendigkeit, den Rezipienten von der gewonnenen Erkenntnis zu überzeugen. Wissenschaftskommunikation ist damit im Kern persuasiv geprägt (vgl. auch Kretzenbacher 1998:135-136). Dieser persuasive Charakter ist untrennbar verknüpft mit einer an der wissenschaftlichen Kontroverse orientierten Verfasstheit dieses Sprachhandlungsbereichs (vgl. Ehlich 1993, Steinhoff 2008, Feilke 2010). So wird Bezug genommen auf andere Positionen, die diskutiert, kritisiert und weitergeführt werden. Auf diese Weise wird es möglich, die eigene Arbeit als einen wichtigen, auf der umfassenden Auseinandersetzung mit dem Forschungsstand basierenden Erkenntniszugewinn erscheinen zu lassen und so die Wissenschaftsgemeinschaft von der erbrachten Leistung zu überzeugen. 
Die sprachlichen Handlungen, welche die Persuasion umsetzen, lassen sich danach unterscheiden, ob ein Dialog mit anderen wissenschaftlichen Forschungspositionen oder mit dem Rezipienten stattfindet. So werden zum einen durch die sprachlichen Handlungen des Zitierens und Verweisens intertextuelle Bezugnahmen auf stützende Forschungspositionen sowie eine Auseinandersetzung mit bzw. Abgrenzung von widersprechenden Ansätzen realisiert. Zum anderen erfolgt durch Metakommentierungen ein Dialog mit dem Rezipienten. Durch sie wird die Gliederung des Textes transparent gemacht, werden die Erwartungen des Lesers gelenkt, bestimmte Interpretationen nahegelegt sowie Ergebnisse bewertet (vgl. Fandrych 2014:96, Fandrych/Graefen 2002:19-20).

Die Realisierung dieser persuasiv geprägten und kontroversenorientierten Sprachhandlungen erfolgt insbesondere durch Mittel der allgemeinen (Schepping 1976) bzw. alltäglichen Wissenschaftssprache (Ehlich 1993). Es handelt sich dabei um disziplinenübergreifend verwendete Ausdrucksmittel, die der Gemeinsprache entstammen und in spezifischer Weise „für die Zwecke der Wissenschaft" (Ehlich 2000:52) genutzt werden. Persuasiv geprägte, kontroversenorientierte Sprachhandlungen lassen sich nicht am einzelnen Lexem festmachen, da sie erst als Mehrwortstrukturen in Erscheinung treten. Daher bieten Wortverbindungskonzepte wie das der Kollokationen einen naheliegenden Ausgangspunkt für die lexikografische Beschreibung der sprachlichen Umsetzung dieser Verwendungsweisen.

\section{Kollokationen als Repräsentationsform allgemeinwissenschaftssprachlicher Handlungen}

Kollokationen werden in lexikografischen Nachschlagewerken üblicherweise zur Veranschaulichung von Lesarten herangezogen. Definiert als nichtidiomatische, auf bestimmte Strukturtypen festgelegte, in der Regel zweigliedrige, konventionalisierte Wortverbindungen (Hausmann 1985, Bahns 1996) ermöglichen sie die Dokumentation der mit einem Lexem und den zugehörigen Lesarten jeweils verbundenen Gebrauchskonventionen. Die Fokussierung auf bestimmte Strukturformen wie Adjektiv-Nomen-, Verb-Nomen- oder auch Adverb-Verb-Verbindungen erlaubt eine Darstellungsweise, durch die typische Verwendungsformen in komprimierter und prägnanter Gestalt präsentiert werden können.

Allerdings wird die Beschränkung auf die Zweigliedrigkeit in neueren Arbeiten weniger restriktiv gehandhabt (u.a. Hausmann 2007:218), da eine Ausweitung auf komplexere Strukturen sowie die semantische Spezifizierung weiterer Kombinationspartner eine präzisere Beschreibung einer Lesart zulässt. Zudem erscheint eine Berücksichtigung des jeweiligen Sprachverwendungsbereichs erforderlich, da Kollokationen hier unterschiedliche Gebrauchsspezifika aufweisen. So konnte 
Wallner (2014) anhand einer Korpusstudie zeigen, dass Kollokationen über ein für die Wissenschaftssprache typisches Gebrauchsprofil verfügen können. Es ist davon auszugehen, dass mittels dieser bereichstypischen Verwendungsmuster wissenschaftsspezifische Kommunikationsbedürfnisse umgesetzt werden.

Aus Perspektive der Lexikografie der allgemeinen Wissenschaftssprache ist eben dieser Handlungsbezug interessant. Es wäre daher zu prüfen, ob sich anhand von Kollokationen die sprachliche Umsetzung des auf die Überzeugung gerichteten Handelns in der Wissenschaftskommunikation lexikografisch darstellen lässt. Dieser Frage soll im Folgenden anhand einer Korpusstudie zur wissenschaftssprachlichen Verwendung der Kollokation Bild zeichnen nachgegangen werden.

\section{Die Kollokation Bild zeichnen im wissenschaftlichen Diskurs: eine empirische Untersuchung}

\subsection{Untersuchungskontext und Datengrundlage}

Die Untersuchung wurde im Rahmen des Projekts GeSIG (Das gemeinsame sprachliche Inventar der Geisteswissenschaften) durchgeführt. Ziel des Projekts ist es, für das Deutsche das Inventar der allgemeinen Wissenschaftssprache geisteswissenschaftlicher Disziplinen auf empirischer Grundlage korpusmethodisch $\mathrm{zu}$ ermitteln und eine Konzeption zu dessen lexikografischer Erschließung zu erarbeiten. Die Datengrundlage für die Ermittlung des Inventars bildet ein Korpus geisteswissenschaftlicher Dissertationen. Dieses setzt sich aus 19 Teilkorpora zusammen. Die Grundlage dafür bildet die Fächereinteilung des statistischen Bundesamtes (2013), in welcher 19 geisteswissenschaftliche Fachdisziplinen unterschieden werden (etwa Geschichte, Romanistik, Philosophie, Musikwissenschaften u.a.). Die Einteilung des Bundesamtes stützt sich auf die institutionelle Organisation des Fachbereichs sowie auf Stellungnahmen der Fachverbände. Jedes Teilkorpus umfasst mindestens 10 Dissertationen und enthält mindestens 1 Million Token. Die Texte wurden nach Wortarten annotiert und lemmatisiert. Auf Grundlage der Teilkorpora wurde für jeden Fachbereich eine Lemmaliste generiert. Das gemeinsame sprachliche Inventar der Geisteswissenschaften wurde durch die Bildung einer Schnittmenge aus den Fachbereichslisten ermittelt. Das Inventar umfasst damit jene sprachlichen Mittel, die der Form nach in geisteswissenschaftlichen Disziplinen übergreifend gebraucht werden. Es bildet den Ausgangspunkt für die Stichwortliste einer digitalen lexikografischen Ressource der allgemeinen Wissenschaftssprache der Geisteswissenschaften. Aktuell wird im Projekt an der Konzeption der lexikografischen Darstellung gearbeitet. Um dem besonderen Charakter der allgemeinen Wissenschaftssprache Rechnung zu tragen, soll der Fokus hierbei auf die Funktionalisierung der sprachlichen Mittel für 
den wissenschaftsmethodologischen Gebrauch gelegt werden. Ein wesentlicher Aspekt ist es zu prüfen, welche komplexeren Strukturen als Beschreibungselemente zur Repräsentation wissenschaftssprachlicher Handlungen herangezogen werden können. In der vorliegenden Untersuchung soll dies anhand des Konzepts der Kollokationen erprobt werden.

Exemplarisch wird dazu die Kollokation Bild zeichnen betrachtet. Diese wurde ausgewählt, da das Lemma Bild zu den häufigsten Nomen des gemeinsamen Inventars zählt (neben Nomen wie etwa Jahr, Form, Text, Frage) und damit dem Kernbereich der allgemeinen Wissenschaftssprache der Geisteswissenschaften angehört. Das Lemma zeichnen ist der häufigste Vollverb-Kookkurrenzpartner von Bild. Mit der Kollokation Bild zeichnen wird so exemplarisch eine quantitativ relevante allgemeinwissenschaftliche Wortverbindung für die Untersuchung herangezogen.

Für die im Folgenden vorgestellte Analyse wurden im gesamten oben beschriebenen Korpus mit Hilfe der Software Antconc 3.4.3 (Anthony 2014) alle Vorkommen der Kollokation Bild zeichnen erhoben. Diese umfassen alle Belege, die Bild und im Abstand von sieben Wörtern (davor oder danach) eine Form des Verbs zeichnen enthalten. Die so erhobenen 157 Vorkommen wurden anschlieBend datengeleitet im Hinblick auf ihre funktionale Verwendung gruppiert. Die folgenden Abschnitte stellen die Ergebnisse der Auswertung vor. Dabei wird zunächst auf das Verwendungsspektrum von Bild zeichnen eingegangen (4.2.). Anschließend wird der Fokus auf qualifizierende Verwendungen dieser Kollokation gelegt, anhand derer sich die sprachliche Umsetzung persuasiv gestalteter Sprachhandlungen verdeutlichen lässt (4.3.).

\subsection{Das Verwendungsspektrum der Kollokation Bild zeichnen}

Für die Kollokation Bild zeichnen zeigen sich in den Daten zwei Lesarten. Dabei handelt es sich zum einen um eine konkrete Bedeutung, bei der es um die Anfertigung eines physisch realen Bildes geht. Belege für diese Lesart (insgesamt 10\% aller Vorkommen) finden sich überwiegend in kunstbezogenen Arbeiten und spiegeln dabei eine durch deren Gegenstandsbereich motivierte Verwendung wider.

(1) Die Konturen der Kreise sind weich und fließend, auf der umliegenden, weißen Fläche zeichnet sich ein zarter, flaumartiger ,Rauchschleier ab. Piene hatte dieses Bild aus freier Hand über Kopf, gezeichnet'. Der Kontrast von Hell und Dunkel erzeugt den Eindruck von Räumlichkeit ... (74_KUGE_2).

Neben diesem konkreten, eher fachbereichsspezifischen Gebrauch findet sich die Kollokation Bild zeichnen auch in abstrakter Lesart zur Versprachlichung einer zusammenfassenden Charakterisierung von Forschungsinhalten. Diese abstrakte 
Lesart manifestiert sich in funktional verschiedenen Verwendungsweisen. Dabei lassen sich die folgenden Typen unterscheiden:

Handlungstyp 1: Verweise auf andere Forschungspositionen und Primärquellen Handlungstyp 2: Evaluierende Charakterisierung von Forschungsdaten Handlungstyp 3: Metakommentierungen

Handlungstyp 4: Formelhafte Formulierung des wissenschaftlichen Anspruchs, der mit einer gewählten Vorgehensweise verbunden wird

Handlungstyp 5: Formulierung von Forschungsaufgaben bzw. -desideraten

Im Folgenden sollen diese Verwendungstypen genauer vorgestellt werden.

\section{Handlungstyp 1: Verweise auf andere Forschungspositionen und Primärquellen}

Am häufigsten (63\% aller Vorkommen) wird die Kollokation Bild zeichnen zur sprachlichen Realisierung von Verweisen eingesetzt. Verweise werden hierbei mit Jakobs (1999:41) verstanden als ,funktional begründete Bezugnahmen von Texten auf andere Texte, die durch sprachliche Handlungen [...] des Verweisens erzeugt und angezeigt werden“. So zeigt Beleg (2) eine Verwendung, mit der auf eine andere Forschungsposition verwiesen wird. An die Stelle des Autors Hans-Günter Funke, auf dessen Arbeit sich der Verweis bezieht, tritt dabei metonymisch seine Definition.

(2) Das Thema der 'Senilità' im Romanwerk Italo Svevos (1979) ist der Titel des Ende der 70er Jahre erschienenen Aufsatzes von Hans-Günter Funke [...]. Seine Definition des inetto zeichnet bereits ein zusammenhängendes Bild jener literarischen Figur, die der Wahl des vorliegenden Textkorpus zugrunde liegt (11_ITAL_4).

Analog zu verweisenden Bezugnahmen auf wissenschaftliche Autoren und ihre Arbeiten finden sich auch Verwendungen, in denen auf Primärtexte bzw. -daten Bezug genommen wird. So zeigt Beleg (3) eine Bezugnahme auf einen literarischen Text, der im Rahmen einer literaturwissenschaftlichen Arbeit aus dem Bereich der Amerikanistik analysiert wird. Anders als die Verweise auf andere wissenschaftliche Texte bzw. Positionen ist diese Form der Bezugnahme nicht unmittelbar Ausdruck der wissenschaftlichen Kontroverse. Vielmehr spiegelt sie die Auseinandersetzung des Autors mit seinem Gegenstand wider.

(3) Plath zeichnet in ihrer Kurzgeschichte das Bild einer überwiegend passiven Frau, die vollkommen im vorherrschenden Weiblichkeitskonzept ihrer Zeit gefangen ist (10_AMERI_3). 


\section{Handlungstyp 2: Evaluierende Charakterisierung von Forschungsdaten}

Am zweithäufigsten (16\% aller Vorkommen) wird die Kollokation Bild zeichnen verwendet, um eine evaluierende Charakterisierung von Forschungsdaten vorzunehmen. Dies zeigt Beleg (4), in dem mit Hilfe der Kollokation Bild zeichnen die Aussagekraft der betrachteten Primärquellen zusammenfassend eingeschätzt wird. Bei Beleg (5) handelt es sich um eine Textstelle, in der die in einer unmittelbar vorangehenden Tabelle aufgeführten Ergebnisse evaluierend kommentiert werden.

(4) Philosophen haben zwar ganze Bücher zur Sprachbetrachtung geschrieben haben (sic!), es ist aber keines ihrer Werke vollständig erhalten. Dennoch läßt sich aus den Fragmenten und der späteren Überlieferung durch Diogenes Laertius ein recht klares Bild ihrer sprachwissenschaftlichen Standpunkte zeichnen (08_ALTGRIECH_3).

(5) Diese Ergebnisse zeichnen ein klares und zugleich kontrastreiches Bild. Wird comme in einem erzählenden Textstück eingesetzt, so kommen in $p$ in etwa zwei von drei Fällen Tempora zum Einsatz, die der Schilderung von Vordergrund dienen (11_FRZ_2).

\section{Handlungstyp 3: Metakommentierungen}

Des Weiteren wird Bild zeichnen (in 8\% aller Vorkommen) zum Ausdruck von Metakommentierungen gebraucht. Die Kollokation findet zur Realisierung von mehreren, bei Fandrych/Graefen (2002) unterschiedenen Textkommentartypen Anwendung. So zeigt Beleg (6) ein Beispiel für die Benennung des Hauptziels der Arbeit, Beleg (7) veranschaulicht die einleitende Qualifizierung einer folgenden Sprechhandlung und Beleg (8) illustriert eine zusammenfassende Selbsteinschätzung in Bezug auf das in der Arbeit Erreichte (vgl. ebd.:21-25).

(6) Um diesen seit fast 2000 Jahren immer wiederkehrenden Vorurteilen entgegen zu treten, soll mit unvoreingenommenem Blick versucht werden, ein weitaus differenzierteres Bild der römischen Gastronomie zu zeichnen als es bisher geschehen ist (05_ARCḦ̈_1).

(7) Aufgrund der Korrelation der Daten zu den Faktoren Lesegewohnheit und familiäre Situation [...] lässt sich folgendes Bild zeichnen: - Die eigenen und familiären Lesegewohnheiten haben einen Einfluss auf die Kategorisierung der Schüler entsprechend ihren bereits erworbenen literaten Fähigkeiten... (09_DAF_3).

(8) Die vorliegende Dissertation zeichnet ebenfalls ein neues Bild Sellners während des Nationalsozialismus und beschreibt ihn als begeisterten Förderer eines neuen nationalsozialistisch geprägten Theaters... (77_ THEATER_3). 


\section{Handlungstyp 4: Formelhafte Formulierung des wissenschaftlichen An- spruchs, der mit einer gewählten Vorgehensweise verbunden wird}

Deutlich seltener (2\% aller Vorkommen) wird die Kollokation Bild zeichnen verwendet, um den wissenschaftlichen Anspruch zu formulieren, dem man mit den eingesetzten Methoden gerecht werden möchte. Beleg (9) zeigt ein Beispiel für diese Verwendungsweise. Es handelt sich dabei um einen Abschnitt, mit dem das Auswertungskapitel eingeleitet wird. Mit Hilfe der Kollokation motiviert die Autorin die zuvor geschilderte Vorgehensweise: Durch die Korrelation des Gebrauchs komplexer NPs mit soziodemografischen Daten soll ein besseres Verständnis der literalen Kompetenzen der Probanden gewonnen werden. Die Kollokation Bild zeichnen fungiert hierbei als Versatzstück, das einen generellen Anspruch wissenschaftlicher Arbeiten ausdrückt, der darin besteht, eine klarere Erkenntnis des Forschungsgegenstandes zu erreichen. Die Kollokationsbasis Bild wird dabei stets mit einem positiv qualifizierenden Adjektiv attribuiert (vgl. 4.3.)

(9) Die (schul-)biografischen, sprachlichen, familiären und sozio-ökonomischen Angaben, die mittels des Interviews erhoben wurden, sollen zur Überprüfung der Hypothesen mit den jeweiligen Ergebnissen des Gebrauchs komplexer NPs der erhobenen Texte korreliert werden. Nach Möglichkeit sollen auf Grundlage des Gebrauchs der komplexen NPs in Erzählungen und Verschriftungen verschiedene Typen gebildet werden, deren Verhalten in Bezug auf literate Strukturen um die sozialen Daten ergänzt werden sollen, um so ein genaueres Bild von den Typen zeichnen zu können (09_DAF_3).

\section{Handlungstyp 5: Formulierung von Forschungsaufgaben bzw. -desideraten}

Schließlich deutet sich ein weiterer Handlungstyp in einigen wenigen Belegen an, in denen die Kollokation Bild zeichnen zur Formulierung von Forschungsaufgaben bzw. -desideraten genutzt wird (1\% aller Vorkommen). Beispiel (10) illustriert diese Verwendung. Es handelt sich dabei um eine Fußnote, in der die Autorin es zur wichtigen Forschungsaufgabe erklärt, die Person Amalie Sieveking aus einer erweiterten Perspektive zu beschreiben. Die Benennung der Forschungsaufgabe wird hier mit Hilfe der Kollokation umgesetzt.

(10) Die Verdienste, die sich Amalie Sieveking mit ihrem Engagement für eine Berufstätigkeit der Frau erworben hat, haben dazu geführt, dass ihre erzieherischen Tätigkeiten weitgehend in den Hintergrund gedrängt worden sind, so dass die Beschaffenheit des Unterrichts, den Caroline [Bertheau] bei ihr genoss, weitgehend im Dunkeln bleibt. Aus diesem Grund erscheint es umso wichtiger, ein über den erzieherischen Bereich hinausgehendes Bild von Amalie Sieveking zu zeichnen (02_DIA_1).

Die Analyse der Belege zeigt, dass die Kollokation Bild zeichnen zur Realisierung verschiedener wissenschaftssprachlicher Handlungen eingesetzt wird. Es ist 
somit keine Eins-zu-eins-Beziehung zwischen Kollokation und Sprachhandlung herstellbar, jedoch wird eine Präferenz für bestimmte Verwendungsweisen deutlich. So wird Bild zeichnen primär zu Versprachlichung von Verweisen herangezogen (63\%). Daneben findet die Kollokation häufiger Anwendung zum Ausdruck einer evaluierenden Charakterisierung von Forschungsdaten (16\%) sowie zur Umsetzung von Metakommentierungen (8\%).

Nachdem eingangs die Wissenschaftskommunikation als persuasiv geprägtes Handlungsfeld eingeführt wurde, sollen im Folgenden die Kollokation Bild zeichnen und die durch sie ausgedrückten wissenschaftssprachlichen Handlungen unter diesem Aspekt betrachtet werden.

\subsection{Qualifizierende Verwendungen der Kollokation Bild zeichnen}

Die persuasive Prägung und die Kontroversenorientiertheit von Wissenschaftskommunikation resultiert, wie oben beschrieben, aus der Notwendigkeit, durch Veröffentlichung die Rezipientengemeinschaft vom Wert der eigenen wissenschaftlichen Ergebnisse zu überzeugen. Die Beurteilung des Werts einer wissenschaftlichen Arbeit erfolgt dabei vor dem Hintergrund globaler wissenschaftlicher Qualitätskriterien, welche Czicza/Hennig (2011:51) unter der Maßgabe einer Orientierung auf Erkenntniszuwachs bündeln. Aller Wissenschaftskommunikation liegt somit „die Ausrichtung auf die Gewinnung und Verbreitung neuer Erkenntnisse“ (ebd.) zugrunde, die sich in ihrer sprachlichen Darstellung widerspiegeln muss.

Bei den mit Hilfe der Kollokation Bild zeichnen realisierten wissenschaftssprachlichen Handlungen ist eine Bezugnahme auf dieses Kriterium besonders deutlich erkennbar, wenn die Attributstelle vor der Kollokationsbasis Bild durch ein qualifizierendes Adjektiv besetzt ist. Eine Analyse der Belegstellen zeigt, dass die Orientierung auf Erkenntniszuwachs in den zu Bild attributiv gebrauchten Adjektiven Niederschlag findet. Durch sie werden verschiedene wiederkehrende stereotype Facetten des Erkenntniszuwachses in ihrer Ausprägung qualifiziert, wobei auf Kategorien wie Detailliertheit, Klarheit, Umfang, Neuheit, Gültigkeit, Konsistenz oder auch Anschaulichkeit rekurriert wird. Während hierbei einerseits auf den Gewinn an wissenschaftlicher Erkenntnis durch eine detailliertere, klarere, umfassendere, neuere oder zutreffendere Einsicht in den Forschungsgegenstand Bezug genommen wird, zielen die Kategorien Konsistenz und Anschaulichkeit stärker auf Gütekriterien wissenschaftlicher Darstellung ab (vgl. Czicza/Hennig 2011:52).

Die genannten Kategorien kommen in den Belegstellen durch die in Tabelle 1 dargestellten Adjektive zum Ausdruck. Es finden sich dabei für das wissenschaftliche Handlungsfeld sowohl positiv als auch negativ konnotierte Formen. Dies zeigt etwa die Kategorie Detailliertheit, deren Ausprägung einerseits durch positiv wertende Adjektivattribute (detailliert, differenziert, vielfältig), andererseits aber auch durch negativ wertende Adjektivattribute (wenig detailliert, wenig differenziert, relativ undifferenziert) versprachlicht wurden. Was hier als auf- bzw. abwer- 
tend verstanden wird, ist aufgrund der Orientierung an wissenschaftlichen Qualitätskriterien für dieses Handlungsfeld spezifisch und nicht unbedingt auf andere Kommunikationsbereiche übertragbar (vgl. etwa die wertschätzende Verwendung des Attributs komplex in der Wissenschaft).

Tabelle 1: Qualifizierende Adjektivattribute zur Kollokation Bild zeichnen

\begin{tabular}{|c|c|}
\hline & ein ... Bild zeichnen \\
\hline Detailliertheit & $\begin{array}{l}\text { detailliert(er)es } \\
\text { differenziert(er)es } \\
\text { vielfältigeres } \\
\text { wenig detailliertes } \\
\text { wenig differenziertes } \\
\text { relativ undifferenziertes }\end{array}$ \\
\hline Klarheit & $\begin{array}{l}\text { klar(er)es } \\
\text { deutliche(re)s } \\
\text { geschärftes } \\
\text { kein einheitliches }\end{array}$ \\
\hline Umfang & $\begin{array}{l}\text { umfassendes } \\
\text { komplexes } \\
\text { einseitiges } \\
\text { unvollständiges }\end{array}$ \\
\hline Neuheit & $\begin{array}{l}\text { neues } \\
\text { über ... hinausgehendes } \\
\text { längst bekanntes }\end{array}$ \\
\hline Gültigkeit & $\begin{array}{l}\text { authentisches } \\
\text { realistischeres } \\
\text { unzutreffendes }\end{array}$ \\
\hline Konsistenz & $\begin{array}{l}\text { konsistentes } \\
\text { halbwegs plausibles } \\
\text { teilweise widersprüchliches }\end{array}$ \\
\hline Anschaulichkeit & plastisches \\
\hline
\end{tabular}

Wie gestaltet sich nun die Funktionalität der qualifizierenden Verwendungen der Kollokation Bild zeichnen für die durch sie realisierten wissenschaftssprachlichen Handlungen im Einzelnen?

Bei den Verweisen (Handlungstyp 1) werden die den genannten Feldern zuordenbaren Adjektivattribute zu Bild herangezogen, um die wissenschaftliche Leistung des referierten Autors zu qualifizieren. So zeigt Beleg (11) eine anerkennende Einschätzung einer wissenschaftlichen Arbeit, die auf die Konsistenz 
als Gütekriterium wissenschaftlicher Darstellung Bezug nimmt. Beleg (12) veranschaulicht hingegen einen kritisierenden Verweis auf eine andere Forscherposition. Die qualifizierende Verwendung der Kollokation Bild zeichnen dient somit einer kontroversenorientierten Darstellung referierter Literatur.

(11) So gelingt es Bailyn (2012) in der Tat, die Daten zu erfassen undmindestens für die genannten Fälle - ein konsistentes Bild zu zeichnen (12_SLAW_1).

(12) Schneider zeichnet ein relativ undifferenziertes Bild davon, dass sich die jungen Filmschaffenden auf die möglichst publikumsunabhängige, künstlerische Ausdrucksform konzentriert hätten und eine deutsche Filmkultur schaffen wollten, die den Film auf eine Ebene mit Kulturinstitutionen wie dem Theater stellt (77_FILM_FERNSEH_4).

Abzugrenzen sind diese kontroversenorientierten Verwendungen von Verweisen, die mit Hilfe der Kollokation gegenstandsbezogene Charakterisierungen vornehmen und damit nicht auf eine Einschätzung der wissenschaftlichen Leistung des referierten Autors abzielen. So zeigt Beleg (13) einen Verweis auf einen als düster charakterisierten Sachverhalt und keine Wertung gegenüber der Arbeit der Autorin Hildenbrand.

(13) Ein ähnlich düsteres Bild von der Feminisierung des Berufsstandes zeichnet die amerikanische Professorin und Bibliothekswissenschaftlerin Suzanne Hildenbrand ... (06_BIBLIO_5).

Daneben sind die kontroversenorientierten Verwendungen zu unterscheiden von in den geisteswissenschaftlichen Belegen mitunter anzutreffenden Verweisen, in denen die literarische Qualität oder Wirkung eines Primärtextes eingeschätzt wird, vgl. Beleg (14). Während erstgenannte disziplinenübergreifend gebraucht werden und damit allgemeinwissenschaftliche Relevanz besitzen, sind die letztgenannten eher von fachspezifischem Interesse.

(14) Einerseits wird dem Leser durch die auftretende Ironie klar gemacht, dass es sich hier um fiktive Texte handelt, andererseits gelingt es dem Erzähler immer wieder, ein authentisches und auch ein dramaturgisch spannendes Bild des damaligen Lebens zu zeichnen ... (11_SPAN_1).

Im Rahmen einer evaluierenden Charakterisierung von Forschungsdaten (Handlungstyp 2) wird die Kollokation Bild zeichnen ebenfalls qualifizierend gebraucht. Auch hier wird durch die eingesetzten Adjektivattribute auf die oben genannten wissenschaftlichen Qualitätskriterien rekurriert. Während in Beleg (15) die betrachteten Primärdaten für die Produktion eines Erkenntniszugewinns als geeignet eingestuft werden, stellt Beleg (16) sie als in dieser Hinsicht unzureichend dar. 
(15) Als Gegenprobe zur deutschen Berichterstattung lassen sich die zahlreichen US-Armeepublikationen heranziehen: Angefangen mit der offiziellen Armeezeitung [...] zu lokalen Zeitschriften und Flugblättern [...] brachten die Armeestellen eine große Menge an Schriftgut hervor, anhand derer sich ein differenziertes Bild des Alltagslebens in der US-Armee zeichnen lässt (05_NEU_1).

(16) Instrumentalschulen bilden wohl den größten Quellenbestand in Bezug auf die Erforschung der Geschichte des instrumentalen Lehrens und Lernens. Sie zeichnen jedoch nur ein unvollständiges Bild der realen Unterrichtssituation [...], weshalb ebenfalls musikhistorische und alltagsgeschichtliche Quellen erforscht werden sollten (78_INSTRU_1).

Bei Metakommentierungen (Handlungstyp 3), die mit Hilfe der Kollokation Bild zeichnen realisiert werden, finden ebenfalls attributive Adjektive Verwendung, die eine Einordnung bezüglich der oben genannten wissenschaftlichen Qualitätskriterien vornehmen. Auffällig ist, dass diese insbesondere bei den Metakommentierungstypen ,Benennung des Hauptziels der Arbeit' sowie der am Ende einer Arbeit vorgenommenen ,zusammenfassenden Selbsteinschätzung' anzutreffen sind. $\mathrm{Zu}$ beobachten ist hierbei, dass die verwendeten Adjektive überwiegend eine positive Qualifizierung vornehmen. So wird in Beleg (6a) das Hauptziel der Dissertation formuliert und durch das Adjektiv differenziert in Hinblick auf den angestrebten Erkenntniszugewinn qualifiziert. Der Gebrauch des Komparativs sowie die Voranstellung der Intensitätspartikel weitaus betonen diesen zusätzlich, indem sie gemeinsam mit dem angeschlossenen Vergleichssatz als es bisher geschehen ist einen Bezug zu bereits vorliegenden Arbeiten herstellen. Beleg (17) zeigt eine in ähnlicher Weise umgesetzte zusammenfassende Selbsteinschätzung. Auch hier wird durch das Adjektiv geschärft eine Qualifizierung in Hinblick auf einen Zugewinn an Erkenntnis vorgenommen, welche durch die vorangestellte Intensitätspartikel deutlich weiter verstärkt wird.

(6a) Um diesen seit fast 2000 Jahren immer wiederkehrenden Vorurteilen entgegen zu treten, soll mit unvoreingenommenem Blick versucht werden, ein weitaus differenzierteres Bild der römischen Gastronomie zu zeichnen als es bisher geschehen ist (05_ARCHÄ_1).

(17) Die Erfassung und Auswertung der technologischen Daten erfolgte im Rahmen der vorliegenden Arbeit anhand einer begrenzten Gruppe von Werken. Es wurde damit möglich ein deutlich geschärftes Bild der Kölner Leinwandbilder zu zeichnen (75_MALEREI_4).

Wird mittels der Kollokation Bild zeichnen der mit einer gewählten Vorgehensweise verbundene wissenschaftliche Anspruch formuliert (Handlungstyp 4), finden 
sich ebenfalls qualifizierende, auf die oben genannten Kategorien wissenschaftlicher Qualität Bezug nehmende Adjektivattribute. Ähnlich wie bei Handlungstyp 2, bei dem Primärdaten im Hinblick auf die Erzielung eines Erkenntniszugewinns eingestuft werden, erfolgt in dieser Hinsicht bei Handlungstyp 4 durch das qualifizierende Adjektivattribut eine Einschätzung der eingesetzten Methoden. Wie bereits beschrieben, wird mittels der Kollokation versatzstückhaft der generelle Anspruch wissenschaftlicher Arbeiten, ein besseres Verständnis des Forschungsgegenstandes zu erreichen, zum Ausdruck gebracht. In Beleg (9a) findet sich dies mit dem Komparativ des Adjektivs genau umgesetzt.

(9a) Nach Möglichkeit sollen auf Grundlage des Gebrauchs der komplexen NPs in Erzählungen und Verschriftungen verschiedene Typen gebildet werden, deren Verhalten in Bezug auf literate Strukturen um die sozialen Daten ergänzt werden sollen, um so ein genaueres Bild von den Typen zeichnen zu können (09_DAF_3).

Auch bei der Formulierung von Forschungsaufgaben und -desideraten (Handlungstyp 5) findet eine Bezugnahme auf einen angestrebten Erkenntniszuwachs statt, der in analoger Weise realisiert wird. In Beleg (10a) wird hierfür das komplexe Attribut über ... hinausgehend genutzt.

(10a) Die Verdienste, die sich Amalie Sieveking mit ihrem Engagement für eine Berufstätigkeit der Frau erworben hat, haben dazu geführt, dass ihre erzieherischen Tätigkeiten weitgehend in den Hintergrund gedrängt worden sind, so dass die Beschaffenheit des Unterrichts, den Caroline [Bertheau] bei ihr genoss, weitgehend im Dunkeln bleibt. Aus diesem Grund erscheint es umso wichtiger, ein über den erzieherischen Bereich hinausgehendes Bild von Amalie Sieveking zu zeichnen (02_DIA_1).

\subsection{Zusammenfassung der Untersuchungsergebnisse}

Es lässt sich festhalten, dass alle Typen der durch die Kollokation Bild zeichnen realisierten wissenschaftssprachlichen Handlungen eine auf den Erkenntniszuwachs abzielende Qualifizierung aufweisen können. Diese wird jeweils durch attributive Adjektive aus den in 4.3. umrissenen semantischen Bereichen sprachlich umgesetzt. Dabei scheint die Auswahl der gebrauchten Adjektive nicht beliebig erweiterbar zu sein, sondern ist begrenzt auf die Versprachlichung stereotyper Facetten des Erkenntniszugewinns, welche durch diese Bereiche repräsentiert werden. Durch den Rückgriff auf diese stereotypen Facetten lassen sich Aspekte der wissenschaftlichen Arbeit plausibilisieren: von Teilschritten bzw. Teilergebnissen, über Vorgehensweisen sowie methodische Ansätze bis hin zu der in einem Beitrag konstatierten wissenschaftlichen Gesamtleistung. Diese Plausibilisierung erfolgt 
mittels einer sprachlich expliziten Qualifizierung, die auf das in der Wissenschaft erwartete Qualitätskriterium des Erkenntniszuwachses rekurriert und durch die sich die Rezipientengemeinschaft vom wissenschaftlichen Wert der erbrachten Leistung überzeugen lässt.

$\mathrm{Zu}$ Beginn des Beitrags wurde die Frage aufgeworfen, wie sich die sprachliche Umsetzung des auf die Überzeugung gerichteten Handelns in der Wissenschaftskommunikation lexikografisch darstellen lässt. Nach der erfolgten exemplarischen Analyse zur Kollokation Bild zeichnen in geisteswissenschaftlichen Dissertationen kann diesbezüglich Folgendes festgehalten werden: Schließt man das adjektivische Attribut zur Kollokationsbasis Bild in die Betrachtung ein, tritt ein übergreifender handlungsbezogener Aspekt hervor. Dieser besteht darin, dass durch die Verwendung von qualifizierenden Adjektiven, die auf einen wissenschaftlichen Erkenntniszuwachs Bezug nehmen, die realisierten Handlungen persuasiv gestaltet werden können. Es hat sich damit auch gezeigt, dass das Kollokationskonzept eine Möglichkeit bietet, die sprachliche Umsetzung persuasiven Handelns in der Wissenschaftskommunikation zu fassen. Es ist jedoch deutlich geworden, dass ein erweitertes Kollokationskonzept zugrunde gelegt werden muss, welches eine Spezifizierung weiterer Kombinationspartner einschließt. So kann die Kollokation Bild zeichnen nur durch die Berücksichtigung der Attributstelle vor der Kollokationsbasis Bild als zu einem persuasiven Handlungscharakter beitragend beschrieben werden. In diesem weiteren Verständnis scheint es somit gerechtfertigt, Kollokationen als lexikografische Beschreibungselemente zur Repräsentation wissenschaftssprachlicher Handlungen heranzuziehen. Im Folgenden soll vor diesem Hintergrund für das Beispiel Bild zeichnen eine mögliche Eintragsstruktur skizziert werden.

\section{Entwurf für eine mögliche lexikografische Darstellung wissenschaftssprachlicher Handlungen}

Der hier vorgestellte Ansatz für eine digitale lexikografische Aufbereitung der allgemeinen Wissenschaftssprache legt den Fokus auf wissenschaftsmethodologisch relevante Verwendungsweisen disziplinenübergreifend gebrauchter Ausdrucksmittel. Für die lexikografischen Einträge ist eine primär gebrauchsbasiert-funktional ausgerichtete Beschreibung vorgesehen. Im Zentrum steht daher weniger die semantische Erläuterung eines Einzellemmas, sondern vielmehr sein funktionaler Gebrauch im Handlungsfeld Wissenschaft. Für jede Lesart eines Lemmas werden daher die vorkommenden sprachlichen Gebrauchsmuster empirisch erfasst und die durch sie realisierten sprachlichen Handlungen ermittelt. Diese bilden den Schwerpunkt der lexikografischen Darstellung.

In der verfolgten Konzeption würde somit die Kollokation Bild zeichnen als ein sprachliches Gebrauchsmuster innerhalb des Eintrags zum Lemma Bild erscheinen. Von ihr ausgehend werden die durch sie realisierten Sprachhand- 
lungen beschrieben. Die Fokussierung auf wissenschaftsmethodologisch relevante Verwendungsweisen hat für die Kollokation Bild zeichnen zur Folge, dass die konkrete Lesart (im Sinne einer Anfertigung eines physisch realen Bildes, vgl. Beleg (1)) keine Berücksichtigung findet, sondern nur die für die abstrakte Lesart unterschiedenen funktionalen Verwendungsweisen einbezogen werden.

Abbildung 1 skizziert eine mögliche lexikografische Darstellung der Kollokation Bild zeichnen. Diese Darstellung umfasst zunächst eine handlungsübergreifende Charakterisierung, welche jene inhaltlichen Aspekte benennt, die in allen durch die Kollokation realisierten wissenschaftsspezifischen Sprachhandlungen anzutreffen sind. Für das Beispiel Bild zeichnen lässt sich diese übergreifende Charakterisierung als Ausdruck einer zusammenfassenden Darstellung und/oder Qualifizierung von Forschungspositionen oder Forschungsinhalten beschreiben. Daran schließt sich die Auflistung jener Sprachhandlungen an, die durch das sprachliche Gebrauchsmuster Bild zeichnen realisiert werden. Dabei beschränkt sich die lexikografische Darstellung auf diejenigen Handlungstypen, die eine ausreichende Häufigkeit und Verbreitung aufweisen. Für die betrachtete Kollokation erscheinen in dieser Hinsicht Verweise (Handlungstyp 1), evaluierende Charakterisierungen von Forschungsdaten (Handlungstyp 2) und Metakommentierungen (Handlungstyp 3) zur Beschreibung geeignet. Für diese erfolgt eine Illustration durch Kontextbelege.

Zusätzlich werden für das Gebrauchsmuster konventionalisierte Verwendungspräferenzen und -restriktionen, die vor allem morphosyntaktische Merkmale betreffen, aufgeführt. Im Fall von Bild zeichnen wäre hier etwa die Beschränkung des nominalen Bestandteils Bild auf den Singular zu nennen.

Wie aus der Analyse hervorgegangen ist, zeigt sich ein übergreifender handlungsbezogener Aspekt in der Verwendung spezifischer adjektivischer Attribute zur Kollokationsbasis Bild. Durch die Verwendung von Adjektiven, die auf das wissenschaftliche Qualitätskriterium des Erkenntniszuwachses Bezug nehmen, ist es möglich, eine auf- oder abwertende Qualifizierung vorzunehmen. Damit konnte ein lexikografisch darstellbares Mittel für die sprachliche Umsetzung persuasiven Handelns in der Wissenschaftskommunikation identifiziert werden. Dieses für alle durch die Kollokation Bild zeichnen realisierten Handlungstypen anwendbare Mittel wird in der lexikografischen Darstellung als „Zusatzinformation zu spezifischen Gebrauchsmöglichkeiten“ ausgewiesen. Hierbei bietet sich für die Darstellung das erweiterte Kollokationskonzept an, welches eine Spezifizierung weiterer Kombinationspartner vorsieht. Im vorliegenden Fall würde dies eine nach Kategorien wissenschaftlicher Qualität (wie Detailliertheit, Klarheit, Umfang u.a.) gruppierte Ausweisung der Besetzungsmöglichkeiten für die Attributstelle zur Kollokationsbasis Bild umfassen. Zur Veranschaulichung der Verwendungsmöglichkeiten werden auch hier Kontextbelege aufgeführt. 


\section{jmd. zeichnet ein $\mathbf{x}_{\text {Attribut }}$ Bild von etw.}

\section{Handlungsübergreifende Charakterisierung}

Ausdruck einer zusammenfassenden Darstellung und/oder Qualifizierung von Forschungspositionen oder

Forschungsinhalten

\section{Durch das Gebrauchsmuster realisierte Sprachhandlungen}

Bestandteil von Verweisen (1), evaluierenden Charakterisierungen von Forschungsdaten (2) und

Metakommentierungen (3)

(1) So gelingt es Bailyn (2012) in der Tat, die Daten zu erfassen und-mindestens für die genannten Fälleein konsistentes Bild zu zeichnen. (SLAWISTIK)

(2) Als Gegenprobe zur deutschen Berichterstattung lassen sich die zahlreichen US-Armeepublikationen heranziehen: Angefangen mit der offiziellen Armeezeitung [...] zu lokalen Zeitschriften und Flugblättern [...] brachten die Armeestellen eine große Menge an Schriftgut hervor, anhand derer sich ein differenziertes Bild des Alltagslebens in der US-Armee zeichnen lässt. (GESCHICHTE)

(3) Aufgrund der Korrelation der Daten zu den Faktoren Lesegewohnheit und familiäre Situation [...] lässt sich folgendes Bild zeichnen:- Die eigenen und familiären Lesegewohnheiten haben einen Einfluss auf die Kategorisierung der Schüler ... (GERMANISTIK)

\section{Gebrauchsspezifika}

Bild nur im Singular belegt

\section{Zusatzinformationen zu spezifischen Gebrauchsmöglichkeiten}

Durch Verwendung der folgenden Attribute zu Bild kann handlungsübergreifend auf das wissenschaftliche Qualitätskriterium des Erkenntniszuwachses Bezug genommen und damit eine auf- oder abwertende Qualifizierung vorgenommen werden (vgl. die Metakommentierung in (4) bzw. den Verweis in (5))

\begin{tabular}{|c|c|c|c|}
\hline \multirow{3}{*}{ ein } & $\begin{array}{l}\text { DETAILLIERTHEIT } \\
\text { detailliert(er)es } \\
\text { differenziert(er)es } \\
\text { vielfältigeres } \\
\text { wenig detailliertes } \\
\text { wenig differenziertes } \\
\text { relativ undifferenziertes }\end{array}$ & \multirow{3}{*}{ Bild } & \multirow{3}{*}{ zeichnen } \\
\hline & $\begin{array}{l}\text { KLARHEIT } \\
\text { klar(er)es } \\
\text { deutliche(re)s } \\
\text { geschärftes } \\
\text { kein einheitliches }\end{array}$ & & \\
\hline & $\begin{array}{l}\text { UMFANG } \\
\text { umfassendes } \\
\text { komplexes } \\
\text { einseitiges } \\
\text { unvollständiges }\end{array}$ & & \\
\hline
\end{tabular}

(4) Um diesen seit fast 2000 Jahren immer wiederkehrenden Vorurteilen entgegen zu treten, soll mit unvoreingenommenem Blick versucht werden, ein weitaus differenzierteres Bild der römischen Gastronomie zu zeichnen als es bisher geschehen ist. (ARCHÄOLOGIE)

(5) Schneider zeichnet ein relativ undifferenziertes Bild davon, dass sich die jungen Filmschaffenden auf die möglichst publikumsunabhängige, künstlerische Ausdrucksform konzentriert hätten und eine deutsche Filmkultur schaffen wollten, die den Film auf eine Ebene mit Kulturinstitutionen wie dem Theater stellt. (FILMWISSENSCHAFT)

Abbildung 1: Entwurf zur lexikografischen Darstellung der Kollokation Bild zeichnen 
Die vorgestellte Eintragsstruktur zeigt in einem ersten Entwurf, wie die sprachliche Umsetzung persuasiven Handelns in der Wissenschaftskommunikation lexikografisch darstellbar gemacht werden kann. Mit der vorgenommenen Analyse konnte durch die Betrachtung der sprachlich expliziten Qualifizierung als eines hierzu gebrauchten Mittels jedoch zunächst erst ein Aspekt beleuchtet werden. Es bleibt Folgeuntersuchungen vorbehalten, weitere Möglichkeiten für die Realisierung des auf Überzeugung gerichteten sprachlichen Handelns in der Wissenschaftskommunikation zu identifizieren, die in einer lexikografischen Aufbereitung der allgemeinen Wissenschaftssprache berücksichtigt werden sollten.

\section{Literatur}

ANTHONY Laurence, 2014, AntConc (Version 3.4.3) [Computer Software], Tokyo, Japan: Waseda University, http://www.laurenceanthony.net/.

BAHNS Jens, 1996, Kollokationen als lexikographisches Problem. Lexicographica Series maior, Tübingen.

CzICZA Dániel / Hennig Mathilde, 2011, Zur Pragmatik und Grammatik der Wissenschaftskommunikation. Ein Modellierungsvorschlag, in: Fachsprache 1-2, S. 36-60.

EHLICH Konrad, 1993, Deutsch als fremde Wissenschaftssprache, in: Jahrbuch Deutsch als Fremdsprache 19, S. $13-42$.

EHLICH Konrad, 2000, Deutsch als Wissenschaftssprache für das 21. Jahrhundert, in: German as a Foreign Language 1, S. $47-63$.

FANDRYCH Christian / GRAEFEN Gabriele, 2002, Text-commenting Devices in German and English Academic Articles, in: Multilingua 21, S. 17-43.

FANDRYCH Christian, 2014, Metakommentierungen in wissenschaftlichen Vorträgen, in: Fandrych C. /Meißner C./Slavcheva A. (Hg.), Gesprochene Wissenschaftssprache: Korpusmethodische Fragen und empirische Analysen, Heidelberg, S. 95-111.

FEILKE Helmuth, 2010, Schriftliches Argumentieren zwischen Nähe und Distanz - am Beispiel wissenschaftlichen Schreibens, in: Àgel V./Hennig M. (Hg.), Nähe und Distanz im Kontext variationslinguistischer Forschung, Berlin/New York, S. 209-231.

HAUSMANN Franz Josef, 1985, Kollokationen in deutschen Wörterbüchern. Ein Beitrag zur Theorie des lexikographischen Beispiels, in: Bergenholtz H./Mugdan J. (Hg.), Lexikographie und Grammatik, Tübingen, S. $118-129$.

HaUSMANN Franz Josef, 2007, Die Kollokationen im Rahmen der Phraseologie. Systematische und historische Darstellung, in: Zeitschrift für Anglistik und Amerikanistik 55/3, S. 217-234.

JAKOBS Eva-Maria, 1999, Textvernetzung in den Wissenschaften. Zitat und Verweis als Ergebnis rezeptiven, reproduktiven und produktiven Handelns, Tübingen.

KRETZENBACHER Heinz, 1998, Fachsprache als Wissenschaftssprache, in: Hoffmann L./Kalverkämper H./Wiegand H.E. (Hg.), Fachsprachen. Handbücher zur Sprach- und Kommunikationswissenschaft (HSK), Berlin/New York, S. 133-142.

ScHEPPING Heinz, 1976, Bemerkungen zur Didaktik der Fachsprache im Bereich des Deutschen als Fremdsprache, in: Rall D./Schepping H./Schleyer W. (Hg.), Didaktik der Fachsprache. Beiträge zu einer Arbeitstagung der RWTH Aachen vom 30.9. bis 4.10.1974, Bonn-Bad Godesberg, S. $13-34$.

Statistisches Bundesamt, 2013, Bildung und Kultur. Studierende an Hochschule - Fächersystematik. Online verfügbar unter: https://www.destatis.de/DE/Methoden/Klassifikationen/BildungKultur/StudentenPruefungsstatistik.pdf. 16.10.2014. 
STEINHOFF Torsten, 2008, Kontroversen erkennen, darstellen, kommentieren. in: Bons I./Gloning, T./Kaltwasser, D. (Hg.), Fest-Platte für Gerd Fritz. Gießen (13 Seiten). Online verfügbar unter: http://www.festschrift-gerd-fritz.de/files/steinhoff_2008_kontroversen_erkennen_darstellen_ und kommentieren.pdf.

WALLNER Franziska, 2014, Kollokationen in Wissenschaftssprachen. Zur lernerlexikographischen Relevanz ihrer wissenschaftssprachlichen Gebrauchsspezifika, Tübingen. 